\title{
Spectroscopy of Planetary Atmospheres and Surfaces with ISO
}

\author{
Bruno Bézard \\ Observatoire de Paris-Meudon, 92195 Meudon cedex, France
}

\begin{abstract}
Spectroscopic observations of Solar-System objects with ISO provided a wealth of new and exciting results. Major findings relevant to planetary atmospheres and surfaces are presented here. Rotational lines of $\mathrm{HD}$ were observed for the first time in the four giant planets, yielding reliable determinations of the $\mathrm{D} / \mathrm{H}$ ratio. The ${ }^{14} \mathrm{~N} /{ }^{15} \mathrm{~N}$ ratio could also be measured in the upper troposphere of Jupiter. Many new hydrocarbons were detected in the giant planets, improving our understanding of the methane photochemistry. In addition, oxygen compounds $\mathrm{H}_{2} \mathrm{O}$ and $\mathrm{CO}_{2}$ ) were detected in their upper atmospheres and in Titan, pointing to exogenic sources of oxygen. Many bands of $\mathrm{SO}_{2}$ ice were observed on Io, providing information on the physical state of this volcanic compound on the surface. Finally, spectra of Mars revealed the presence of a few so-far undetected mineralogical features, tentatively attributed to carbonates.
\end{abstract}

\section{Introduction}

During its lifetime (1995-1998), the Infrared Space Observatory ISO ${ }^{1}$ observed the planets from Mars to Pluto, satellites of Jupiter and Saturn, a few comets, and several asteroids. Compared to previous space or ground-based observations, the strength of ISO comes from the access to a large infrared range from 2.4 to $240 \mu \mathrm{m}$, the high sensitivity of all four instruments, and the good spectral resolution of the short- and long-wavelength spectrometers (SWS and LWS). Most of the results presented here derive from observations with the SWS, operating in the 2.3-45 $\mu \mathrm{m}$ range with a resolving power of 1500-3000 in the grating mode and $\approx 30000$ in the Fabry-Pérot mode. On the other hand, the aperture (from $14 \times 20^{\prime \prime}$ to $20 \times 33^{\prime \prime}$ ) did not allow for spatially-resolved observations except marginally for Jupiter.

ISO observations of planets and satellites cover the solar radiation reflected by surfaces and atmospheric clouds, the thermal emission emitted by surfaces and atmospheric compounds, and, in a few cases, fluorescence from the upper planetary atmospheres.

\footnotetext{
${ }^{1}$ ISO is an ESA project with instruments funded by ESA Member States (especially the PI countries: France, Germany, the Netherlands, and the United Kingdom) with the participation of NASA and ISAS.
} 


\section{Isotopic ratios in the giant planets}

\section{1. $\mathrm{D} / \mathrm{H}$}

Rotational lines of HD were observed by ISO in the four giant planets. Their analysis, combined with those of the $\mathrm{S}(0)$ and $\mathrm{S}(1)$ quadrupolar lines of $\mathrm{H}_{2}$, yields the $\mathrm{HD} / \mathrm{H}_{2}$ abundance ratio and thus directly the $\mathrm{D} / \mathrm{H}$ ratio in the atmosphere (Feuchtgruber et al. 1999; Lellouch et al. 2001). Prior to ISO, this determination was based on the $\mathrm{CH}_{3} \mathrm{D} / \mathrm{CH}_{4}$ abundance ratio and was thus dependent on some assumption on the fractionation between methane and hydrogen.

The D/H ratio inferred for Jupiter and Saturn $\left(\sim 2 \times 10^{-5}\right)$ agrees with that measured in situ by the Galileo probe and with the protosolar value derived from solar wind measurements. These two planets are predominantly made of hydrogen and helium so that the $\mathrm{D} / \mathrm{H}$ ratio should reflect that of the gaseous component of the solar nebula. On the other hand, Uranus and Neptune are strongly enriched in $\mathrm{C}, \mathrm{N}, \mathrm{O}$ compounds, originally in the form of D-rich ices. The present $\mathrm{D} / \mathrm{H}$ ratio in their atmospheres $\left(\sim 6 \times 10^{-5}\right)$ is higher than the protosolar value. Using most recent interior models, it is possible to trace back to the deuterium abundance in the proto-Uranian and -Neptunian ices. The derived value, bearing large uncertainties, appears similar to those measured in longperiod comets $\left(\sim 3 \times 10^{-4}\right)$. This result agrees with the current assumption that comets from the Oort cloud have a composition similar to the icy planetesimals that formed Uranus' and Neptune's cores.

\section{2. ${ }^{14} \mathrm{~N} /{ }^{15} \mathrm{~N}$}

Weak lines from the ${ }^{15} \mathrm{~N}$ isotope of ammonia were detected by ISO in Jupiter. A combined analysis of these lines with those of ${ }^{14} \mathrm{NH}_{3}$ provided a measurement of the ${ }^{14} \mathrm{~N} /{ }^{15} \mathrm{~N}$ ratio in the upper troposphere of the planet (around 400 mbar). The result, about twice the protosolar value, is surprising. It may mean that the planetesimals that accreted to form the proto-Jupiter were depleted in ${ }^{15} \mathrm{~N}$ with respect to the bulk of the nebula. This could be linked to the trapping of nitrogen in water clathrates at low temperature on which very little is known. Another possibility is that this isotopic anomaly is due to fractionation processes at work in Jupiter's atmosphere. It is possible that ammonia condensation enriches the solid phase and depletes the gas phase in the heavier isotope, thus increasing the ${ }^{14} \mathrm{~N} /{ }^{15} \mathrm{~N}$ ratio in the atmosphere above the condensation level ( $\sim 700$ mbar). Laboratory measurements are definitely needed to better understand this measurement.

\section{Photochemistry}

\subsection{Hydrocarbon photochemistry}

Methane $\left(\mathrm{CH}_{4}\right)$ and its main photochemical products ethane $\left(\mathrm{C}_{2} \mathrm{H}_{6}\right)$ and acetylene $\left(\mathrm{C}_{2} \mathrm{H}_{2}\right)$ have been observed by infrared spectroscopy for over 20 years (except on Uranus). ISO detected for the first time a series of new compounds: the methyl radical $\left(\mathrm{CH}_{3}\right)$ on Saturn and Neptune, acetylene on Uranus, ethylene $\left(\mathrm{C}_{2} \mathrm{H}_{4}\right)$ on Neptune, methyl-acetylene $\left(\mathrm{CH}_{3} \mathrm{C}_{2} \mathrm{H}\right)$ on Jupiter and Saturn, diacetylene $\left(\mathrm{C}_{4} \mathrm{H}_{2}\right)$ on Saturn, and benzene $\left(\mathrm{C}_{6} \mathrm{H}_{6}\right)$ on Jupiter and Saturn. 
The detection of $\mathrm{C}_{2} \mathrm{H}_{2}$ on Uranus, combined with the non-detection of $\mathrm{CH}_{4}$, allowed us to set precise constraints on the eddy mixing rate in the stratosphere and confirmed its sluggishness. $\mathrm{CH}_{3}$ is the first radical ever observed in a planetary atmosphere. Methyl radicals are directly produced from methane photolysis and are the sole source of ethane. Their detection may provide constraints on the eddy mixing near the methane homopause, provided that a lab measurement of the $\mathrm{CH}_{3}$ recombination rate at low temperature can be made. The first firm observation of $\mathrm{C}_{2} \mathrm{H}_{4}$ on Neptune with the ISO photometer led to a column density much lower than predicted by previous photochemical modelling. Its abundance is very sensitive to the $\mathrm{CH}_{4}$ photolysis branching ratio to $\mathrm{CH}$ and can thus help to discriminate between various photolysis schemes proposed in the literature. Finally, it should be noted that benzene is not yet incorporated in photochemical models as the chemical scheme leading to its formation at low temperature $(150-200 \mathrm{~K})$ and pressure $(<1 \mathrm{mbar})$ is unknown. More details and references pertaining to these observations are given in Bézard (2000).

\subsection{Oxygen compounds}

The detection by ISO of water vapor in the stratospheres of the four giant planets was unexpected on thermochemical grounds: abundant in the troposphere, water freezes out at the cold tropopause. $\mathrm{H}_{2} \mathrm{O}$ was also detected on Titan with a corresponding oxygen influx about half that on Saturn. External fluxes implied by the observations are similar on all planets, especially on Jupiter, Saturn and Titan $\left(\sim 10^{6}-10^{7} \mathrm{O}\right.$-atoms $\left.\mathrm{cm}^{-2} \mathrm{~s}^{-1}\right)$. This suggests that micrometeoroid ablation, either from interplanetary dust (due to cometary activity) or interstellar dust, is an important contributor. Precise photochemical and diffusion calculations for Saturn (Moses et al. 2000) confirm that this is likely.

In addition, a signature from $\mathrm{CO}_{2}$ was seen on Jupiter, Saturn, and Neptune. Supply of $\mathrm{CO}_{2}$ from micrometeoroid ablation alone is not sufficient, especially for Saturn. More likely is a photochemical origin. Photolysis of $\mathrm{H}_{2} \mathrm{O}$ produces $\mathrm{OH}$ radicals that can react with hydrocarbons to form $\mathrm{CO}$. Reaction of $\mathrm{OH}$ with $\mathrm{CO}$ then produces $\mathrm{CO}_{2}$. On Jupiter, the limited spatial resolution of the ISO measurements indicates that $\mathrm{CO}_{2}$ is concentrated in the southern hemisphere and not detectable at high northern latitudes. This peculiar distribution suggests that the presence of $\mathrm{CO}_{2}$ is a remnant of the collision of comet Shoemaker-Levy 9 that struck Jupiter in July 1994 near $45^{\circ} \mathrm{S}$ latitude. The cometary impacts deposited large amounts of $\mathrm{CO}$ and $\mathrm{H}_{2} \mathrm{O}$ in the upper atmosphere. As predicted by post-SL9 evolution models, carbon dioxide is subsequently produced by the reaction of $\mathrm{CO}$ with $\mathrm{OH}$ radicals.

\section{Planetary surfaces}

\subsection{Io}

Io's spectrum on the leading hemisphere, recorded by ISO/SWS between 2.4 and $5.3 \mu \mathrm{m}$, shows many bands from $\mathrm{SO}_{2}$ ice with an unprecedented level of details. The precise location, shape and intensity of these bands are functions of the temperature of the frost, the grain size distribution, the spatial coverage on the disk, and dilution with other molecules. This analysis requires precise 
spectroscopic measurements of $\mathrm{SO}_{2}$ ice in various conditions as well as radiative transfer modelling, both currently in progress.

\subsection{Mars}

The spectrum of Mars, recorded in the full range 2.4-45 $\mu \mathrm{m}$, shows with a high $\mathrm{S} / \mathrm{N}$ ratio bands from atmospheric $\mathrm{CO}_{2}, \mathrm{H}_{2} \mathrm{O}$ and $\mathrm{CO}$ (Lellouch et al. 2000). No new gas species were detected.

Besides atmospheric features, the spectrum shows various signatures from the surface. In the solar reflected region, the strong hydration band extending from 2.7 to $3.7 \mu \mathrm{m}$ is prominent. It is reasonably well reproduced by phyllosilicates or basalts. At longer wavelengths, the thermal emission spectrum shows spectral features that cannot be attributed to gaseous absorption. The broad 8$12 \mu \mathrm{m}$ absorption is the well known silicate signature and probably results from dust particles of basaltic composition suspended in the atmosphere. A couple of remaining features at 7.2 and $11.1 \mu \mathrm{m}$ cannot be accounted for by silicates and are tentatively attributed to carbonates. The closest match to the data is achieved by calcite although the band positions do not perfectly agree. If confirmed, this detection would be extremely important since carbonate rocks are believed to be the primary sink of carbon dioxide in the early Martian atmosphere.

\section{Conclusion}

Spectroscopic observations with ISO provided great advancements in various areas of planetary sciences. While some measurements still await to be fully understood (often in need of challenging laboratory work), infrared astronomers are looking ahead to new opportunities, one of the most promising being the Far Infra-Red and Submillimetre Telescope (FIRST) in less than a decade.

\section{References}

Bézard, B. 2000, ESA-SP, 456, 3

Feuchtgruber, H., Lellouch, E., Bézard, B., Encrenaz, T., de Graauw, T., \& Davis, G.R. 1999, A\&A, 341, L17

Lellouch, E., Encrenaz, T., de Graauw, T., Erard, S., Morris, P., Feuchtgruber, H., Crovisier, J., Girard, T., \& Burgdorf, M. 2000, Planet. Space Sci, 48, 1393

Lellouch, E., Bézard, B., Fouchet, T., Encrenaz, T., Feuchtgruber, H., \& de Graauw, T. 2001, A\&A, 370, 610

Moses, J.I., Lellouch, E., Bézard, B., Gladstone, G.R., Feuchtgruber, H., \& Allen, M. 2000, Icarus, 145, 166 